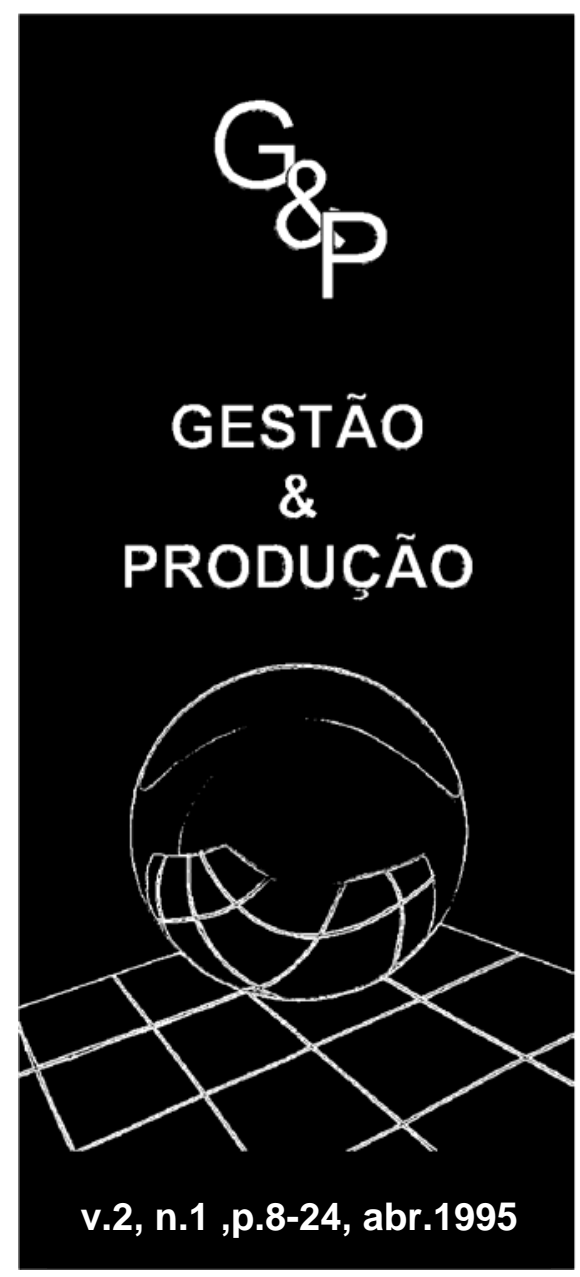

\title{
PLANEJAMENTO AGREGADO DA PRODUÇÃO ÓTIMO COM LIMITE MÍNIMO DE ESTOQUE INFLUENCIADO PELAS INCERTEZAS DE DEMANDA
}

\author{
Oscar S. Silva Filho \\ Marcius F. H. Carvalho \\ Moisés M. Henrique \\ Carlos A. Fernandes \\ Fundação Centro Tecnológico Para Informática \\ Instituto de Automação \\ Caixa Postal 6162 \\ 13081-970 - Campinas / SP \\ Email: oscar@ia.cti.br
}

\section{Resumo}

$O$ artigo trata da determinação de uma política ótima de decisão para um problema de planejamento da produção com restrições de estoque e produção. $O$ horizonte de planejamento é finito, aproximadamente de 1 a 2 anos, com período de discretização mensal. Os dados do problema estão totalmente agregados e a flutuação de demanda ao longo dos períodos do horizonte é aleatória, com distribuição de probabilidade assumida como gaussiana. Assim, o problema estudado é de planejamento estocástico com restrição probabilística na variável de estoque. Mostra-se que é possível, a partir de transformações apropriadas, obter uma formulação determinística equivalente, para a qual uma solução do tipo malha-aberta (que é uma solução aproximada para o problema original) pode ser gerada. É também mostrado que as incertezas relacionadas com flutuações futuras de demanda são explicitadas na formulação determinística por meio de uma função restrição para o limite mínimo do nível de estoque. Esta função é essencialmente côncava e crescente e depende da variância da variável de estoque e de uma medida de probabilidade, fixada a priori pelo usuário. Para ilustrar os desenvolvimentos teóricos, um exemplo simples de um sistema de produção do tipo monoproduto é proposto e resolvido por meio de programação dinâmica determinística. Então, a solução malha-aberta (i.e. solução aproximada) gerada pelo problema equivalente é comparada com a solução verdadeira do problema estocástico, obtida via algoritmo de programação estocástica. 
Este artigo considera o problema de encontrar uma política ótima de produção para uma equação de balanço de estoque, que evolui ao longo de períodos discretos de tempo, dentro de um horizonte de plane-jamento finito. O problema é formulado como um problema de planejamento da produção, do tipo estocástico, com a restri-ção de serem nãonegativas as variáveis nível de estoque e nível de produção. O critério a ser minimizado consiste de custos esperados de armazenagem e de produção, ambos considerados como quadráticos, seguindo assim o modelo proposto por Holt, Modigliani, Muth e Simon - HMMS (HAX, 1978). A demanda pelo produto é descrita como uma variável aleatória com distribuição de probabilidade gaussiana, conhecida para todos os períodos do horizonte de planejamento. Como conseqüência imediata da aleatoriedade da demanda, tem-se que a dinâmica linear do processo pro-dutivo (i.e., a equação de balanço de esto-que) deve ser entendida como um processo estocástico com distribuição gaussiana. Isto significa que a variável de estoque é uma variável aleatória que, ao longo do tempo, comporta-se segundo um processo gaussiano. Deste fato segue que é impossível garantir que, para um dado período de tempo, o nível de estoque não venha a violar a restrição de não-negatividade imposta ao problema. Para reduzir este risco, dois enfoques são propostos na literatura (LASSERE et al., 1985), a saber: um consiste na penalização de possíveis violações diretamente no critério; enquanto o outro procura manter explicitamente a restrição, considerando-a para isto em probabilidade, ou seja, ela passa a ser uma restrição de chance, como é denominada em programação matemática estocástica (VAJDA, 1972). Neste estudo, optou-se pela adoção do último enfoque pois, como será visto adiante, é possível obter uma representação determinística equivalente para a restrição, levando em conta a natureza linear-gaussiana do sistema produtivo.

O problema estocástico assim formulado atende a uma ampla classe de problemas reais encontrados na prática. Do ponto de vista da hierarquia clássica de planejamento (HAX, 1978), ele se enquadra no âmbito das decisões estratégicas, com horizonte de planejamento envolvendo 1 a 2 anos. Neste âmbito tem-se interesse em investigar mais a relação entrada-saída de um processo produtivo e seus limites, ou seja, como entrada têm-se os níveis de produção e como saída, os níveis de estoque (produtos acabados) que são produzidos pelo sistema produtivo em períodos mensais, para atender uma demanda flutuante. O problema apresenta-se aqui com um alto nível de agregação dos dados, ou seja, produtos similares são agrupados em famílias de produtos, enquanto o sistema produtivo (agrupamento de máquinas) tem sua capacidade de produção previamente estabelecida para cada família de produtos. As soluções encontradas servem como informações adicionais para que os gerentes possam estabelecer os chamados planos de produção (HILL, 1991), que irão servir como guias na execução de planejamentos mais detalhados para a empresa. Apesar de que o grau de agregação dos dados reduz bastante o número de variáveis do problema, o número de famílias distintas de produtos, mantém-se, em geral, elevado e, conseqüentemente, resolver o problema estocástico, via programação dinâmica estocástica, é uma tarefa inviável do ponto de vista computacional e a natureza estocástica do problema torna impraticável o emprego de algoritmos de programação matemática.

Para contornar as dificuldades acima, propõe-se uma solução determinística para o problema estocástico, transformado-o em um problema determinístico equivalente. $\mathrm{O}$ critério quadrático e a natureza lineargaus-siana do sistema garantem que estas trans-formações possam ser executadas 
sem grandes dificuldades teóricas e ainda garan-tem a preservação de propriedades estru-turais do problema original, tais como: a convexidade do critério e a linearidade do sistema e das restrições.

A transformação da restrição probabilística gera um limite mínimo de estoque que é dado por uma função côncava crescente, que depende do valor da medida de probabilidade para a restrição probabilística, fixada pelo usuário, e da variância da variável de estoque. Dependendo do valor da medida de probabilidade, a função restrição poderá ser positiva ou negativa. Agora, como os clientes em geral não aceitam atrasos freqüentes nas encomendas, torna-se necessário escolher uma medida de probabilidade que mantenha a função res-trição sempre positiva.

Modelos de sistemas de produção empregando restrições probabilísticas, no sentido de garantir que variáveis de estoque e produção não violem limites preestabe-lecidos, em decorrência de demanda esto-cástica, vêm sendo bastante explorados na literatura em diferentes tipos de aborda-gens, por exemplo: LASSERE et al. (1985] analisa um modelo não-linear para proble-mas de tamanho de lote com restrição probabilística na restrição nível de estoque; FEIRING \& SASTRI (1991) estudam os efeitos da flutuação de demanda estocástica sobre os níveis de estoque e produção, empregando para isso um modelo de pro-gramação linear com restrição probabilís-tica sobre a dinâmica linear do sistema; (PARLAR, 1984) emprega métodos da teoria de controle para gerar planos de produção a partir da solução de problemas de controle ótimo contínuo no tempo com restrições probabilísticas; e (SILVA Fo. et al., 1994) apresenta um método em duas etapas para tratar um problema de plane-jamento da produção estocástica com res-trições probabilísticas nas variáveis de esto-que e de produção.

As seções seguintes deste trabalho estão distribuídas como segue: a seção 2 apresenta a formulação do problema estocástico, juntamente com sua notação e ressalta alguns aspectos importantes desta formulação; a seção 3 discute a transformação do problema estocástico em um problema determinístico equivalente e analisa aspectos da função restrição limite de estoque; a seção 4 apresenta a aplicação do algoritmo de programação dinâmica ao problema determinístico equivalente, para o caso mo-noproduto, e analisa algumas características da solução ótima; e, finalmente, a seção 5 apresenta um exemplo de um sistema de produção multiestágio, monoproduto. A utilização deste exemplo visa aplicar o algoritmo de programação dinâmica para resolver os dois problemas e, assim, poder comparar as duas políticas de decisão ge-radas, ou seja, a política ótima aproximada com a política ótima verdadeira.

\section{Planejamento da Produção: enfoque estocástico}

Seja o seguinte problema:

$$
\underset{u(k)}{\operatorname{Min}} J(u)=h \cdot \sum_{k=0}^{N} E\left\{x(k)^{2}\right\}+c \cdot \sum_{k=0}^{N} u(k)^{2}
$$

s.a.

$$
\left.\begin{array}{r}
x(k)=x(k-1)+u(k)-v(k) ; \operatorname{com} x_{0} \text { e } x_{N} \text { dados } \\
\operatorname{Pr}\{x(k) \geq 0\} \geq 1-\alpha \\
u(k) \geq 0
\end{array}\right\} \mathrm{k}=1,2, \ldots, N
$$


com a seguinte notação:

$\mathrm{x}(\mathrm{k})$ = nível de estoque no período $\mathrm{k}$.

$\mathrm{u}(\mathrm{k})$ = nível de produção no período $\mathrm{k}$.

$\mathrm{v}(\mathrm{k})$ = nível aleatório de demanda no período $\mathrm{k}$, com média $\overline{\mathrm{X}}(\mathrm{k})$ e desvio $\sigma_{\mathrm{v}} \geq 0$.

$\mathrm{h} \cdot \mathrm{x}(\mathrm{k})^{2}=$ custo de estoque no instante $\mathrm{k}$; $\mathrm{h}>0$.

$\mathrm{c} \cdot \mathrm{u}(\mathrm{k})^{2}=$ custo de produção no instante $\mathrm{k}$; $\mathrm{c}>0$.

$\mathrm{x}(0)=\mathrm{x}_{0}$ é o estoque inicial.

$\mathrm{x}(\mathrm{N})=\mathrm{x}_{\mathrm{N}}$ denota o estoque final.

$\mathrm{N}$ = número de períodos (define o horizonte de planejamento).

= medida de probabilidade.

$\mathrm{E}\{\}=$ operador esperança matemática.

O objetivo de (1) é determinar uma seqüência de decisões $\{\mathrm{u}(1), \mathrm{u}(2), \ldots, \mathrm{u}(\mathrm{N})\}$ que minimize os custos médios de estoque e produção, num horizonte finito de planejamento, satisfazendo as restrições.

Neste problema as demandas $\{\mathrm{v}(1)$, $\mathrm{v}(2), \ldots, \mathrm{v}(\mathrm{N})\}$ são variáveis aleatórias independentes e não-negativas com distribuição de probabilidade conhecida, para cada período, neste caso assumidas como gaussianas.

Uma vez que as flutuações aleatórias da demanda afetam a dinâmica da equação de balanço de estoque, a variável de estoque $\mathrm{x}(\mathrm{k})$, para cada período, passa a ser também uma variável aleatória, com distribuição de probabilidade gaussiana, devido à linearidade da equação dinâmica. Por seu turno, o nível de produção $\mathrm{u}(\mathrm{k})$ não depende do nível de demanda observado no período $\mathrm{k}$, conseqüentemente $\mathrm{u}(\mathrm{k})$ é uma variável determinística para todos os períodos do horizonte $\mathrm{N}$.

As variáveis de estoque e produção estão restritas a assumirem valores não-negativos. Assim, a restrição $\mathrm{x}(\mathrm{k})$ signi-fica que a demanda de cada período deve ser totalmente atendida, ou seja, não são admitidos atrasos na entrega de produtos (backlogging). A restrição $\mathrm{u}(\mathrm{k}) \quad 0$ indica que não é fixado um limite máximo para a capacidade de produção, significando que o sistema produtivo tem capacidade de processar grandes quantidades de produtos, em cada período k. Note-se ainda que, sendo a variável de estoque aleatória, não é possível considerar a restrição $\mathrm{x}(\mathrm{k})$ di-retamente em (1); assim, optou-se por considerar esta restrição em probabilidade, com a medida de probabilidade fixada pelo usuário. Por exemplo, se o usuário seleciona $=.05$, significa que as chances de atender completamente a demanda (i.e. satisfação do cliente), em cada período $\mathrm{k}$, serão de pelo menos $95 \%$.

O critério adotado em (1) consiste de custos quadráticos tanto para os níveis de estoque quanto para os níveis de produção. $\mathrm{O}$ emprego de critérios quadráticos permite penalizar valores positivos e negativos das variáveis de estoque e produção, além de ser fácil de manipular, principalmente quando o estudo envolve variáveis aleatórias, como é o caso em (1), onde deve ser calculado o valor esperado do critério, isto é, a média (primeiro momento estatístico) para cada período k do horizonte de planejamento.

Por fim, o problema (1) é definido como um problema de planejamento da produção do tipo estocástico com restrições nas variáveis de estoque e produção. Derivar uma política ótima em malha-fechada, ou seja, uma política de produção que depende do nível de estoque observado em cada período (NECK, 1984), para este problema é uma tarefa complicada, exceto em casos muito simples (por exemplo: monoproduto) com o emprego do algoritmo de programação dinâmica. Soluções aproximadas (como é o caso de soluções em malha-aberta, em que a política de produção gerada depende somente do nível de estoque observado no período inicial $k=0$ (NECK, 1984)) são em geral a melhor opção para tratar sistemas de grande dimensão (sistemas multiproduto) (LASSE-RE et al., 1984). A seção seguinte mostra a transformação de (1) em um equivalente determinístico de mais fácil solução. 


\section{Planejamento da Produção: enfoque determinístico}

O propósito desta seção é converter o problema (1) em um problema determinístico equivalente. Antes de iniciar o processo de transformação é importante considerar que a função distribuição de probabilidade para a variável de estoque
$\left(\mathrm{F}_{\mathrm{X}, \mathrm{k}}, \mathrm{k}\right)$ é inteiramente determinada a partir das equações da média e da variância, as quais são inferidas da equação de balanço de estoque em (1), e estão apresen-tadas a seguir:

Equação da Média:

$$
\overline{\mathrm{x}}(\mathrm{k})=\overline{\mathrm{x}}(\mathrm{k}-1)+\overline{\mathrm{u}}(\mathrm{k})-\overline{\mathrm{v}}(\mathrm{k}) ; \quad \overline{\mathrm{x}}(0)=\mathrm{x}_{0}
$$

Equação da Variância:

$$
\sigma_{\mathrm{x}}^{2}(\mathrm{k})=\mathrm{k} \cdot \sigma_{\mathrm{v}}^{2} ; \quad \sigma_{\mathrm{x}}^{2}(0)=0
$$

onde

$\overline{\mathrm{x}}(\mathrm{k})=\mathrm{E}\{\mathrm{x}(\mathrm{k})\}$ denota a média da variável de estoque;

$\sigma_{\mathrm{x}}^{2}(\mathrm{k})=\mathrm{E}\left\{(\mathrm{x}(\mathrm{k})-\overline{\mathrm{x}}(\mathrm{k}))^{2}\right\}$ denota a variância da variável de estoque; e $\overline{\mathrm{u}}(\mathrm{k})=\mathrm{u}(\mathrm{k})$ indica que a variável de produ-ção é determinística (i.e., $\left.\sigma_{\mathrm{u}}^{2}(\mathrm{k})=0\right)$.
$\mathrm{E}\{\mathrm{x}(\mathrm{k}-1) \cdot \mathrm{v}(\mathrm{k})\}=\mathrm{E}\{\mathrm{x}(\mathrm{k}-1)\} \cdot \mathrm{E}\{\mathrm{v}(\mathrm{k})\}$ não há correlação temporal entre as variáveis de estoque e demanda.

Conseqüentemente, $F_{x, k}$ é perfeitamente caracterizada para cada período $\mathrm{k}$ do horizonte de planejamento e tem a forma:

$$
F_{x, k}(a)=\frac{1}{\sqrt{2 \pi} \cdot \sigma_{x}(k)} \cdot \int_{-\infty}^{a} \exp \cdot\left(-\frac{1}{2}\left[\frac{x(k)-\bar{x}(k)}{\sigma_{x}(k)}\right]^{2}\right) \cdot d x(k)
$$

Além disso, a função (4) é bijetora, o valor “a” tem-se: que significa dizer que para um arbitrário

$$
\mathrm{F}_{\mathrm{x}, \mathrm{k}}(\mathrm{a})=\operatorname{Pr}(\mathrm{x} \leq \mathrm{a}) \geq \alpha \Leftrightarrow \mathrm{a} \geq \mathrm{F}_{\mathrm{x}, \mathrm{k}}^{-1}(\alpha)
$$

onde $F_{x, k}^{-1}($.$) denota a função distribuição de$ probabilidade inversa.
Assim, tem-se que (4) e (5) são importantes propriedades para realizar as transformações desenvolvidas a seguir.

\section{(i) Transformação do Critério:}

É imediato de (4) que

$$
\begin{aligned}
E\left\{x(k)^{2}\right\} & =\int_{-\infty}^{+\infty} x(k)^{2} \cdot d F_{x, k} \\
& =\bar{x}(k)^{2}+\sigma_{x}^{2}(k) \\
& =\bar{x}(k)^{2}+k \cdot \sigma_{v}^{2}
\end{aligned}
$$


e $E\left\{\mathrm{u}(\mathrm{k})^{2}\right\}=\overline{\mathrm{u}}(\mathrm{k})^{2}$, pois a variável de pro- dução é determinística.

\section{(ii) Transformações na restrição de probabilidade:}

Seja a seguinte mudança de variável:

$$
\varepsilon_{\mathrm{x}}(\mathrm{k})=\frac{\mathrm{x}(\mathrm{k})-\overline{\mathrm{x}}(\mathrm{k})}{\sigma_{\mathrm{x}}(\mathrm{k})} \sim \mathrm{N}(0,1)
$$

onde $\mathrm{x}$ denota o resíduo e $N(0,1)$ representa a distribuição de probabilidade nor-

mal com média zero e desvio padrão unitário. Como conseqüência segue que:

$$
\begin{aligned}
\operatorname{Pr}(\mathrm{x}(\mathrm{k}) \geq 0) & =\operatorname{Pr}\left(\overline{\mathrm{x}}(\mathrm{k})+\sigma_{\mathrm{x}} \varepsilon_{\mathrm{x}}(\mathrm{k}) \geq 0\right) \\
& =\operatorname{Pr}\left(\varepsilon_{\mathrm{x}}(\mathrm{k}) \geq-\frac{\overline{\mathrm{x}}(\mathrm{k})}{\sigma_{\mathrm{x}}(\mathrm{k})}\right) \\
& =1-\operatorname{Pr}\left(\varepsilon_{\mathrm{x}}(\mathrm{k}) \leq-\frac{\overline{\mathrm{x}}(\mathrm{k})}{\sigma_{\mathrm{x}}(\mathrm{k})}\right) \geq 1-\alpha \\
& =1-\mathrm{F}_{\varepsilon, \mathrm{k}}\left(-\frac{\overline{\mathrm{x}}(\mathrm{k})}{\sigma_{\mathrm{x}}(\mathrm{k})}\right) \geq 1-\alpha
\end{aligned}
$$

A partir da propriedade (5) aplicada a

(8) obtém-se que

$$
\overline{\mathrm{X}}(\mathrm{k}) \geq-\sigma_{\mathrm{x}}(\mathrm{k}) \cdot \mathrm{F}_{\varepsilon, \mathrm{k}}^{-1}(\alpha) \geq-\sqrt{\mathrm{k}} \cdot \sigma_{\mathrm{v}} \cdot \mathrm{F}_{\varepsilon, \mathrm{k}}^{-1}(\alpha)=\mathrm{r}_{\alpha}(\mathrm{k})
$$

onde $r(k)$ denota a função limite mínimo de estoque (ou estoque de segurança). É importante notar que esta função é diretamente proporcional a $\sqrt{\mathrm{k}}, \sigma_{\mathrm{v}}$, e a $\mathrm{F}_{\mathrm{x}}^{-1}(\alpha)$. Isto produz importantes efeitos sobre $\mathrm{o}$ comportamento de $\mathrm{r}(\mathrm{k})$, a saber:

a) o contorno da função $\mathrm{r}$ (k) é definido pela raiz quadrada do período $\mathrm{k}$, que é uma função côncava e crescente, o que torna a função restrição também côncava e crescente, como ilustrado na figura 1 ;

b) a proporcionalidade com o desvio padrão da demanda significa que o grau de incerteza quanto ao comportamento futuro da demanda é projetado sobre a restrição de estoque e esta incerteza cresce com o passar dos períodos, mediante a evolução da variância do estoque (3), que atinge seu valor máximo no último período do horizonte de planejamento $\mathrm{N}$, isto é, $\sigma_{\mathrm{x}}^{2}(\mathrm{~N}) \geq \sigma_{\mathrm{x}}^{2}(\mathrm{k}) \geq \sigma_{\mathrm{x}}^{2}(\mathrm{k}-1), \forall \mathrm{k} \in[1, \mathrm{~N}] \quad$ (ver figura 1.(a)). Assim, quanto maior for o va- lor de $\sigma_{\mathrm{v}}$, maior será o estoque de segurança nos períodos finais, assegurando assim que as incertezas quanto às flutuações da demanda não irão provocar uma insuficiên-cia nos níveis de estoque, capaz de comprometer o atendimento da demanda (stockout), principalmente nos períodos próximos do final do horizonte de tempo;

c) a medida de probabilidade $\quad 0,1)$, fixada pelo usuário, tem um importante efeito sobre a função $r(\mathrm{k})$. Para entender tal efeito é necessário antes lembrar que a função distribuição de probabilidade normal inversa $\mathrm{F}_{\varepsilon, \mathrm{k}}^{-1}(\alpha)$ tem a seguinte característica: para valores de fixados no intervalo $[0,1 / 2)$ a função $F_{\varepsilon, k}^{-1}(\alpha) \quad 0$; por outro lado, quando $\quad[1 / 2,1)$ o valor desta função será não-negativo. Com base nisto, a função $r(\mathrm{k})$ pode ser interpretada sob dois enfoques distintos, como indicado abaixo:

$$
\begin{array}{lllllll}
\text { Enfoque 1) } & \mathrm{r}(\mathrm{k}) & 0 & \text { (estoque de segurança), } & \text { se } & {[0,1 / 2)} \\
\text { Enfoque 2) } & \mathrm{r}(\mathrm{k}) & 0 & \text { (backlogging), } & \text { se } & {[1 / 2,1)}
\end{array}
$$


O enfoque 1) é o de interesse, neste estudo, uma vez que ele garante o nível médio de estoque (9) sempre positivo. Em outras palavras, ele cria um estoque de segurança capaz de satisfazer as flutuações reais da demanda ao longo de todos os períodos do horizonte de planejamento, o que garante os prazos de entrega do produto ao cliente, aumentando assim o nível de confiança deste pela empresa. Por outro lado, o enfoque 2), não é realista neste estudo, pois seu emprego significa a possibilidade de violar a restrição de não-negatividade imposta pelo problema original (1). Entretanto, ele pode ser interpretado eco-nomicamente como uma facilidade de ne-gociação, entre a gerência de vendas da empresa e o cliente, onde este último consente atrasos na entrega dos produtos encomendados. A função $r(k)$ para $o$ enfoque 2) é convexa e crescente.

Duas importantes propriedades da função restrição $r$ (k) são:

Propriedade 1) Para um dado valor $\sigma_{\mathrm{v}} 0$, a área de segurança (ver figura

(a)

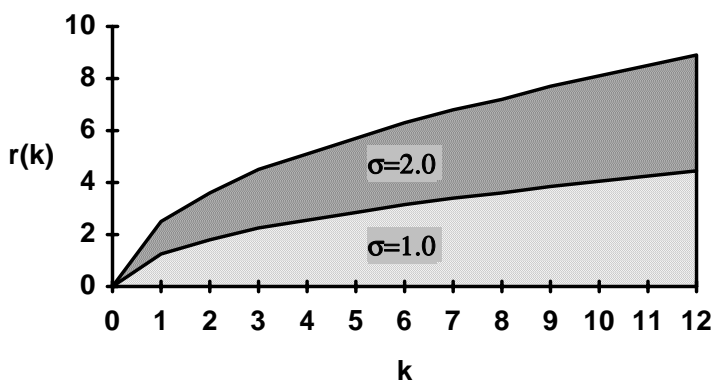

1.(b)) gerada pela função $r$ (k) cresce à medida que decresce. Assim, sejam $\alpha_{1}$ e $\alpha_{2}$ me-didas de probabilidade, fixadas pelo usuário e pertencentes ao intervalo $[0,1 / 2)$ Então, se $\alpha_{1} \leq \alpha_{2} \Rightarrow r_{\alpha_{1}}(k) \geq r_{\alpha_{2}}(k), \forall k \in[0, N]$.

Propriedade 2) Para $\sigma_{\mathrm{v}}=0$ ou para = $1 / 2\left(\right.$ com $\left.\sigma_{\mathrm{v}} 0\right)$, tem-se que $\mathrm{r}(\mathrm{k})=0$. No primeiro caso, desde que o desvio padrão da demanda seja nulo, as flutuações de demanda são inteiramente conhecidas e o problema (1) é determinístico, não fazendo sentido manter a restrição de não-negatividade para o estoque em probabilidade. No segundo caso, ao fixar em 1/2, o usuário admite, com $50 \%$ de chance, que em algum período $\mathrm{k}$ não haja estoque suficiente para atender a demanda.

Figura 1. Estoque de Segurança: (a) $=0.1$ fixo com $\sigma_{v}=1.0$ e $\sigma_{v}=2.0$;

$$
\text { e (b) } \sigma_{\mathrm{v}}=2.0 \text { fixo com }=0.1 \text { e }=0.3 \text {. }
$$

Por fim, tomando-se por base as transformações (2), (6) e (9), o problema deter- minístico equivalente associado a (1) pode ser formulado como segue:

$$
\operatorname{Min}_{\overline{\mathrm{u}}_{\mathrm{k}}} \mathrm{J}(\mathrm{u})=\mathrm{h} \sum_{\mathrm{k}=0}^{\mathrm{N}} \overline{\mathrm{X}}(\mathrm{k})^{2}+\mathrm{c} \sum_{\mathrm{k}=0}^{\mathrm{N}} \overline{\mathrm{u}}(\mathrm{k})^{2}+\mathrm{K}
$$

s.a.

$$
\begin{aligned}
& \overline{\mathrm{x}}(\mathrm{k})=\overline{\mathrm{X}}(\mathrm{k}-1)+\overline{\mathrm{u}}(\mathrm{k})-\overline{\mathrm{v}}(\mathrm{k}) ; \quad \overline{\mathrm{x}}(0)=\mathrm{x}_{0} \\
& \left.\begin{array}{c}
\overline{\mathrm{x}}(\mathrm{k}) \geq \mathrm{r}_{\alpha}(\mathrm{k}) \\
\overline{\mathrm{u}}(\mathrm{k}) \geq 0
\end{array}\right\} \mathrm{k}=1,2, \ldots, \mathrm{N}
\end{aligned}
$$

onde 


$$
\begin{aligned}
& \mathrm{K}=\text { constante }=\mathrm{h} \cdot \sum_{\mathrm{k}=0}^{\mathrm{N}} \mathrm{k} \cdot \sigma_{\mathrm{v}}^{2} \equiv \mathrm{h} \cdot\left(\left(\mathrm{N}^{2}+\mathrm{N}\right) / 2\right) \cdot \sigma_{\mathrm{v}}^{2} \\
& \mathrm{r}_{\alpha}(0)=0 ; \mathrm{e} \\
& \alpha \in[0,1 / 2) .
\end{aligned}
$$

Deve-se salientar que (10) mantém as mesmas propriedades estruturais do proble-ma (1), tais como: a convexidade do crité-rio, a linearidade da dinâmica e restrições de desigualdade nas variáveis de estoque e produção, só que neste caso são determi-nísticas.
Além disso, este problema pode ser resolvido por meio de técnicas modernas da teoria de controle ótimo (PARLAR, 1984) e de programação matemática (AVRIEL, 1976), sendo que esta última permite a solução de problemas de grande dimensão (multiproduto) (LASSERE, 1985).

\section{Solução Ótima via Programação Dinâmica}

Em problemas de produção do tipo monoproduto (ou seja, aqueles que envolvem uma série de produtos agregados em uma única família), pode-se estudar a solução ótima malha-aberta do problema (10) via aplicação do algoritmo de programação dinâmica (PD). Além disso, é possível determinar a solução ótima verdadeira (malha-fechada) do problema proposto em (1), por meio da versão estocástica do algoritmo PD. Isto permite a aferição do modelo proposto em (10), uma vez que é possível comparar as duas soluções gera-das. É importante acrescentar que o emprego de PD em problemas do tipo multiproduto não é possível, em virtude da chamada maldição da dimensionalidade (BERTESEKAS, 1976; NECK, 1984), que diz que o tempo computacional requerido para resolver problemas de grande porte é totalmente impraticável. A seguir discute-se a aplicação do algoritmo PD ao problema (10) e analisam-se algumas características de sua solução ótima.

A forma clássica adotada pela técnica PD para assegurar que a restrição $\bar{x}(k) \geq r_{\alpha}(k)$ não seja violada em um dado período $\mathrm{k} \quad[0, \mathrm{~N}]$, é restringir a variável nível de produção $\overline{\mathrm{u}}(\mathrm{k})$ a adotar valores de um conjunto $\Omega(\overline{\mathrm{x}}(\mathrm{k}-1)) \neq \varnothing$, que depende do nível de estoque remanescente do período k-1, isto é, estoque disponível no início do período k. Para o problema (10), este conjunto é definido como segue: considere-se inicialmente a restrição de não-negatividade, imposta pelo modelo, à variável nível de produção, ou seja, $\bar{u}(\mathrm{k})$ 0. Em seguida, partindo-se da restrição (9), ou seja,

$$
\overline{\mathrm{x}}(\mathrm{k})=\overline{\mathrm{x}}(\mathrm{k}-1)+\overline{\mathrm{u}}(\mathrm{k})-\overline{\mathrm{v}}(\mathrm{k}) \geq \mathrm{r}_{\alpha}(\mathrm{k})
$$

obtém-se a seguinte desigualdade:

$$
\overline{\mathrm{u}}(\mathrm{k}) \geq \mathrm{r}_{\alpha}(\mathrm{k})+\overline{\mathrm{v}}(\mathrm{k})-\overline{\mathrm{x}}(\mathrm{k}-1)
$$

da qual se conclui que

$$
\overline{\mathrm{u}}(\mathrm{k}) \in \Omega(\overline{\mathrm{x}}(\mathrm{k}-1))=\left\{\overline{\mathrm{u}} \mid \overline{\mathrm{u}}(\mathrm{k})=\max \left(0, \mathrm{r}_{\alpha}(\mathrm{k})+\overline{\mathrm{v}}(\mathrm{k})-\overline{\mathrm{x}}(\mathrm{k}-1)\right)\right\}
$$


Conhecida a restrição (12), pode-se escrever a equação recursiva do algoritmo de PD, a qual será resolvida backward no tempo, isto é, a primeira iteração ocorrerá no período $\mathrm{N}-1$ e a última no período 0 :

$$
\begin{gathered}
\mathrm{J}_{\mathrm{N}}(\overline{\mathrm{x}}(\mathrm{N}))=\mathrm{h} \overline{\mathrm{x}}(\mathrm{N})^{2} \\
\mathrm{~J}_{\mathrm{k}}(\overline{\mathrm{x}}(\mathrm{k}))=\operatorname{Min}_{\overline{\mathrm{u}}(\mathrm{k}) \in \Omega}\left\{\left(\mathrm{h} \overline{\mathrm{x}}(\mathrm{k})^{2}+\mathrm{c} \overline{\mathrm{u}}(\mathrm{k})^{2}\right)+\mathrm{J}_{\mathrm{k}+1}(\overline{\mathrm{x}}(\mathrm{k}+1))\right\} \\
\mathrm{k}=0,1,2, \ldots, \mathrm{N}-1
\end{gathered}
$$

O nível ótimo de produção, para cada período k, obtido das iterações de (13) é dado por:

$$
\overline{\mathrm{u}}(\mathrm{k})=\mu_{\mathrm{k}}^{*}(\overline{\mathrm{x}}(\mathrm{k}-1)), \quad \mathrm{k} \in[1, \mathrm{~N}]
$$

onde $\mu($.$) denota o operador ótimo que$ indica que $\overline{\mathrm{u}}(\mathrm{k})$ depende do nível de estoque do período k-1. Deve-se notar que embora $\overline{\mathrm{u}}(\mathrm{k})$ dependa da medida de estoque $\overline{\mathrm{x}}(\mathrm{k}-1)$, a política ótima de produção (14) é do tipo malha-aberta, o que é explicado pelo fato de o problema (10) ser determinístico. O custo total de operação do sistema (estoque + produção) é obtido no último estágio de iteração do algoritmo (13), para um dado estoque inicial $\overline{\mathrm{x}}(0)$, isto é, $\mathrm{J}^{*}=\mathrm{J}_{0}\left(\overline{\mathrm{x}}_{0}\right)$.

\section{Características da solução ótima:}

a) a solução ótima (14) e o custo de operação obtido dependem implicitamente da medida de probabilidade , por meio da restrição (12). De fato, desde que o valor de , como visto na seção anterior (propriedade 1), influi na área formada pelo contorno da função restrição $r_{\alpha}(k)$ (ver figura 1.(b)), o conjunto sofrerá também essa influência, restringindo mais ou menos o nível de produção para diferentes valores de . b) a partir do espaço de produção (12), as seguintes condições podem ser deduzidas sobre o comportamento da solução ótima (14), a saber:

(b.1) Nada se produz, em um período $k$, isto é, $\overline{\mathrm{u}}(\mathrm{k})=0$.

se para algum $\mathrm{k} \quad[1, \mathrm{~N}]$, ocorrer que $r_{\alpha}(k)+\bar{v}(k)-\bar{x}(k-1) \leq 0$, tem-se que $\overline{\mathrm{u}}(\mathrm{k})=0$. Conseqüentemente, a condição (b.1) existirá somente se o nível de estoque no início do período k satisfizer a seguinte desigualdade:

$$
\overline{\mathrm{x}}(\mathrm{k}-1) \geq \mathrm{r}_{\alpha}(\mathrm{k})+\overline{\mathrm{v}}(\mathrm{k})
$$

Esta característica é observada principal-mente quando o sistema produtivo opera com um estoque inicial bastante elevado (BENSOUSSAN et al., 1983).

(b.2) Produz-se, em um período k, uma quantidade $\overline{\mathrm{u}}(\mathrm{k})>0$ se para algum $\mathrm{k} \quad[1, \mathrm{~N}]$, ocorrer que $\mathrm{r}_{\alpha}(\mathrm{k})+\overline{\mathrm{v}}(\mathrm{k})-\overline{\mathrm{x}}(\mathrm{k}-1)>0$, tem-se que $\overline{\mathrm{u}}(\mathrm{k})>0$. Conseqüentemente, a condição (b.2) só será verificada se o nível de estoque no início do período $\mathrm{k}$ satisfizer a seguinte desigualdade: 


$$
\overline{\mathrm{x}}(\mathrm{k}-1)<\mathrm{r}_{\alpha}(\mathrm{k})+\overline{\mathrm{v}}(\mathrm{k})
$$

Além disso, desde que para todo $\mathrm{k}$ é imposto que $\bar{x}(k) \geq r_{\alpha}(k)$, tem-se que existe uma região factível, definida pela faixa que tem como limite inferior o contorno da cur-va $\mathrm{r}_{\alpha}(\mathrm{k})$ e como limite superior o contorno da curva formada por $r_{\alpha}(k)+\bar{v}(k)$, na qual a variável $\overline{\mathrm{x}}(\mathrm{k})$ poderá adotar valores. Por absurdo, suponha-se que sob a condição (b.2), exista um $\overline{\mathrm{u}}(\mathrm{k})=0$, neste caso pode-se verificar, relacionando a equação (2) com a desigualdade (16), que o estoque resultante do período $\mathrm{k}$, que estará disponível no instante $\mathrm{k}+1$, será $\overline{\mathrm{x}}(\mathrm{k})<\mathrm{r}_{\alpha}(\mathrm{k})$, violando assim o limite mínimo estabelecido pela função restrição $r_{\alpha}$.
Ainda com relação à condição (b.2) é possível mostrar que o nível de estoque $\overline{\mathrm{X}}(\mathrm{k})$ ao tocar, uma única vez, na função restrição $r_{\alpha}(k)$ é por ela absorvido e mantém-se neste estado até o final do horizonte de planejamento N (PARLAR, 1985). Esta característica da solução só será violada se o estado final $\overline{\mathrm{X}}(\mathrm{N})$ tiver sido fixado a priori. A seguir discute-se o efeito desta característica sobre a solução ótima (14).

Supondo-se que no início do período $\mathrm{k}$, $\overline{\mathrm{x}}(\mathrm{k}-1)=\overline{\mathrm{r}}_{\alpha}(\mathrm{k}-1)$, então, a partir de (2) e (9), segue que:

$$
\begin{gathered}
\overline{\mathrm{u}}(\mathrm{k})=\mathrm{r}_{\alpha}(\mathrm{k})+\overline{\mathrm{v}}(\mathrm{k})-\mathrm{r}_{\alpha}(\mathrm{k}-1) \quad \Leftrightarrow \\
\overline{\mathrm{u}}(\mathrm{k})=(\sqrt{\mathrm{k}}-\sqrt{\mathrm{k}-1}) \sigma_{\mathrm{v}} \mathrm{F}_{\mathrm{x}, \mathrm{k}}^{-1}(\alpha)+\overline{\mathrm{v}}(\mathrm{k}) \\
\overline{\mathrm{u}}(\mathrm{k})=\Delta \mathrm{r}_{\alpha}(\mathrm{k})+\overline{\mathrm{v}}(\mathrm{k})
\end{gathered} \Leftrightarrow
$$

O resultado dado em (17), significa que para garantir que $\overline{\mathrm{x}}(\mathrm{k})=\mathrm{r}_{\alpha}(\mathrm{k})$, no período $\mathrm{k}$, torna-se necessário produzir o nível de demanda no período $\overline{\mathrm{v}}(\mathrm{k})$ e adicionar a variação incremental da função $r_{\alpha}$, entre os períodos k-1 e $\mathrm{k}$, aqui denotada por $\Delta \mathrm{r}_{\alpha}(\mathrm{k})$. Para os períodos seguintes $k+1, k+2, \ldots, N$, a política de produção seguirá (17).

\section{Exemplo de Aplicação}

Considere a situação de uma determinada firma, onde as vendas de um único produto (ou em um contexto mais amplo de uma família de produtos similares) no horizonte de tempo de um ano, estão sujeitas a flutuações mensais de demanda, definidas por uma distribuição gaussiana, com médias (mensais) e desvios (iguais) conhecidos a partir dos históricos de vendas.

O objetivo da gerência é determinar um plano anual de produção que forneça subsídios para a realização do planejamento detalhado da firma (i.e., aquele que envolve decisões de médio e curto prazo).

O modelo de otimização determinístico formulado em (10) pode ser empregado para gerar o plano. Este modelo, além de ser de fácil solução do ponto de vista computacional, permite ao gerente analisar várias situações que podem afetar o plano de produção, bastando para isto que alguns parâmetros do modelo sejam manipulados, como é o caso da condição inicial x(0) e da medida de probabilidade .

A seguir, emprega-se o exemplo para testar algumas das propriedades discutidas na seção 3, quando da transformação de (1) no modelo equivalente (10). E também para mostrar algumas das características da solução ótima de (10) apresentadas na seção 4. Neste sentido, três casos serão analisados a seguir, sendo que o último deles é dedicado a comparar a solução óti- 
ma verdadeira (obtida de (1) via PD estocástica) com a solução ótima aproximada de (10).
Os dados do problema monoproduto estão listados na tabela 1.

Tabela 1. Dados do Problema

\begin{tabular}{|l|l|l|l|l|l|l|l|l|l|l|l|l|}
\hline mês k & Jan & Fev & Mar & Abr & Mai & Jun & Jul & Ago & Set & Out & Nov & Dez \\
\hline $\mathrm{v}(\mathrm{k})$ & 7 & 8 & 7 & 7 & 6 & 4 & 5 & 4 & 5 & 5 & 6 & 8 \\
\hline \multicolumn{1}{c|}{$\sigma_{\mathrm{v}}^{2}=2.0$} & $\mathrm{~N}=12$ \\
\hline
\end{tabular}

\section{CASO 1: Efeito da variação do estoque inicial sobre a solução do problema}

Para estudar este efeito, fixa-se a medida de probabilidade em 0.05 (i.e., 95\% de chances de atendimento da demanda). Analisam-se então duas situações (cená-rios), a saber: o primeiro cenário considera um estoque inicial elevado, e no segundo adota-se um estoque inicial relativamente pequeno.

Cenário 1.1: $x_{0}=30$.
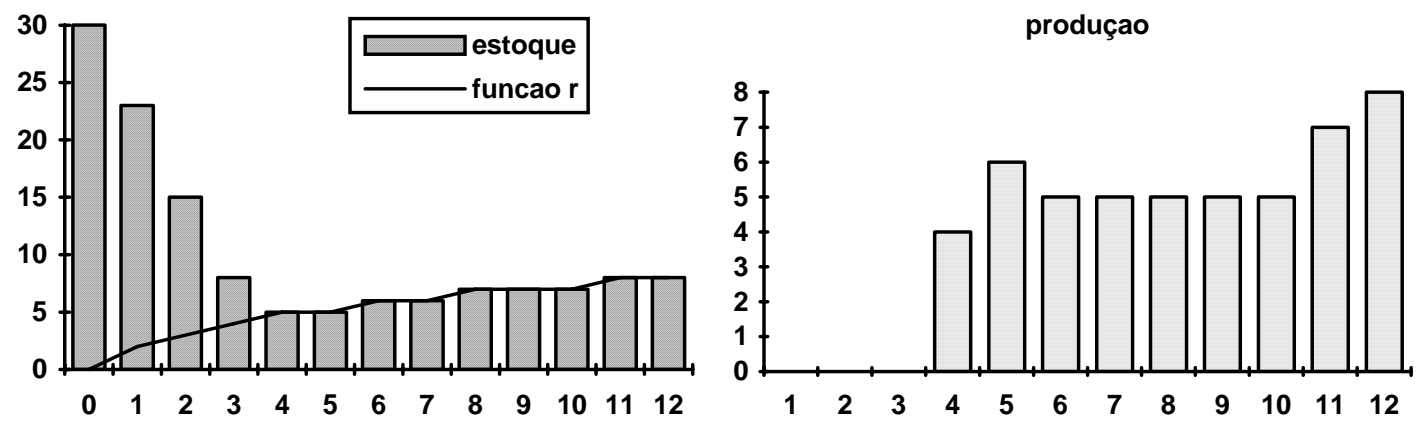

Figura 2

A figura 2 mostra os níveis ótimos de estoque e produção. Dela pode-se observar que enquanto o nível de estoque, nos períodos iniciais ( $\mathrm{k}=1$,2 e 3), é maior que o nível de demanda acrescido do nível de estoque de segurança, dado por $r_{\alpha}$, para

$$
\mathrm{x}_{0}>>(1 / 12) \cdot \sum_{\mathrm{k}=1}^{12} \overline{\mathrm{v}}(\mathrm{k})=6.0
$$

obtida da tabela 1. Além deste resultado pode-se ainda observar que, no quarto período $(k=4)$, o nível de estoque toca a função limite $r_{\alpha}$ e é por ela absorvido, permanecendo neste estado até o final do cada um destes períodos, nada é produzido (vide condição (b.1), seção 4). Isto ocorre devido ao fato de o nível de estoque inicial $\left(\mathrm{x}_{0}=30\right)$ ser elevado em relação à demanda média anual, isto é: horizonte de planejamento $(\mathrm{N}=12)$. Esta característica da solução ótima foi discutida na condição (b.2) da seção 4, na qual se mostrou que a partir do momento em que o estoque é absorvido, o nível 
ótimo de produção (14) segue a equação

Cenário 1.2: $x_{0}=5$
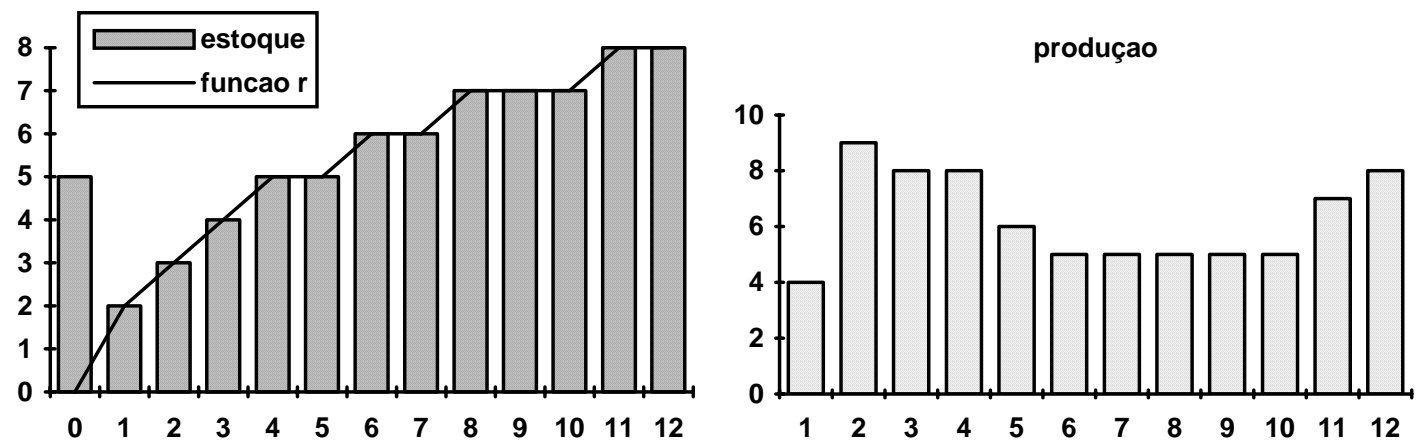

Figura 3

Em contraste com o cenário anterior, o estoque inicial agora é menor que a deman-da média anual $\left(\mathrm{x}_{0}<6\right)$. Conseqüente-mente, o nível de estoque é imediatamente absorvido pela função limite $\mathrm{r}_{\alpha}$, já no pe-ríodo $\mathrm{k}=1$, e o nível ótimo de produção se-gue (17). A figura 3 apresenta os níveis óti-mos de estoque e produção para este caso.

Comentário: Uma vez que para o exemplo em questão, o custo de estoque $(\mathrm{h}=2)$ é o dobro do custo de produzir ( $c=1$ ), ter um estoque inicial muito elevado pode significar prejuízos para a empresa. Isto pode ser constatado comparando-se os custos obtidos para os cenários 1.1 e 1.2. No primeiro obteve-se $\mathrm{J}^{*}(30)=4.788$ enquanto no segundo $\mathrm{J}^{*}(5)=1.569$, o que significa com- parativamente um custo adicional de $205 \%$.

A título de ilustração, considera-se novamente a situação do cenário 1.1 , só que supondo-se agora que o estoque final não é livre, seu valor é, por exemplo, $x(13)=30$. Neste caso então, como se pode observar na figura 4, o nível de estoque é forçado, nos dois últimos períodos, a abandonar a função $r_{\alpha}$. Como conseqüência desta operação, o custo torna-se bastante elevado $\left(\mathrm{J}^{*}\left(\mathrm{x}_{0}=30\right)=7.328\right)$. Comparan-do aos custos anteriores, chega-se a um custo adicional de $53 \%$ em relação ao custo obtido no cenário 1.1 e de $367 \%$ em relação ao custo no cenário 1.2 .
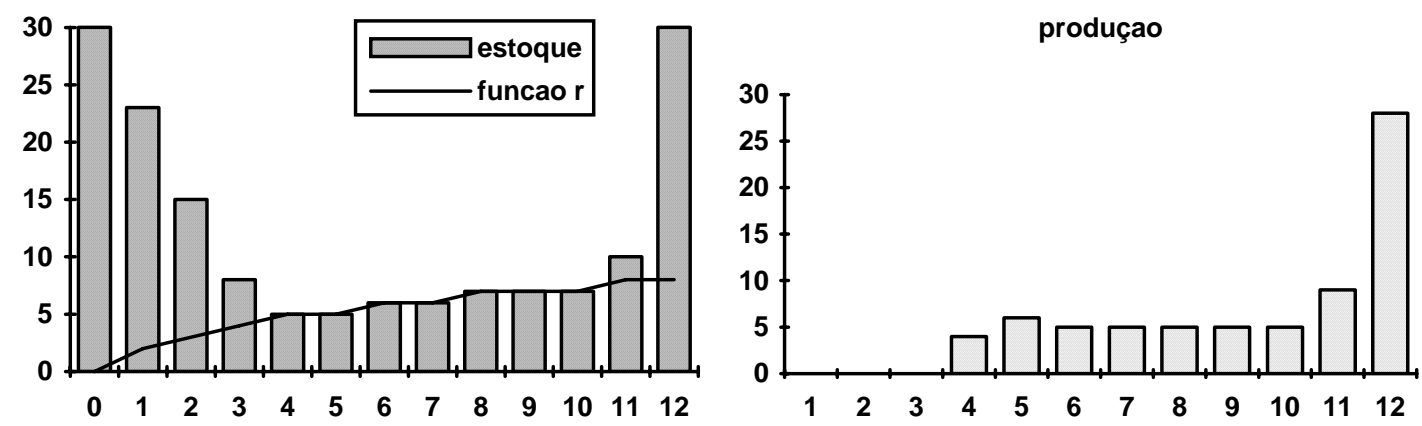

Figura 4.

\section{CASO 2: Efeito de diferentes valores de sobre a solução do problema}


Analisam-se três cenários para 1/2]. O nível de estoque inicial é mantido

\section{Cenário 2.1: $\quad=0.05$}

A figura 5 apresenta os níveis de estoque e produção obtidos para este cenário, o fixo $\left(\mathrm{x}_{0}=15\right)$ e o estoque final é livre. Segue então:

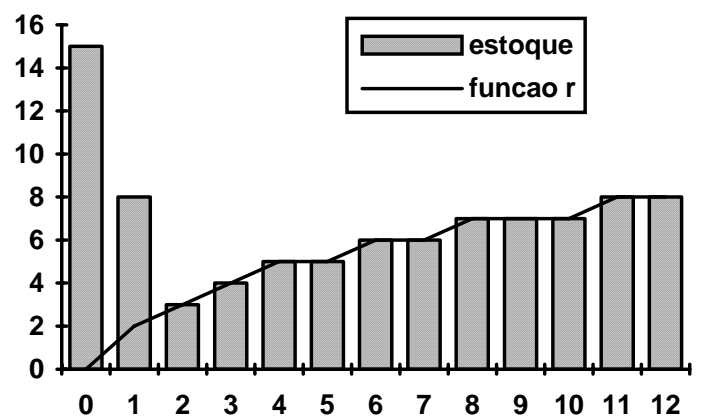

custo total da operação do sistema foi de $\mathrm{J}^{*}=2.019$.

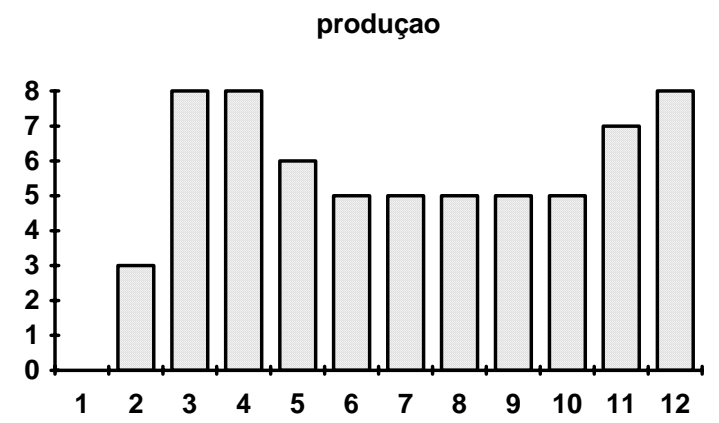

Figura 5

\section{Cenário 2.2: $=0.25$}

O custo total foi de $\mathrm{J}^{*}=1.378$, e os níveis de estoque e produção são apresentados na figura 6.


\section{Cenário 2.3: $=0.5$}

Neste caso, em particular, $\mathrm{r}_{\alpha}(\mathrm{k})=0$ para todos os períodos $k$, ou seja, despreza-se $o$ estoque de segurança e, conseqüentemen-te, quando a política ótima de produção é aplicada ao sistema de produção sujeito às flutuações futuras de demanda, podem ocor-rer atrasos na entrega do produto (ver pro-priedade 2,

Figura 6.

seção 3). Em termos práticos, o gerente da firma acredita na possibilidade de negociar com o cliente possíveis atrasos na entrega do produto. O custo ótimo obtido para este tipo de operação é de $\mathrm{J}^{*}=1.221$ e os níveis ótimos de produção e estoque estão apresentados na figura 7 . 
estoque

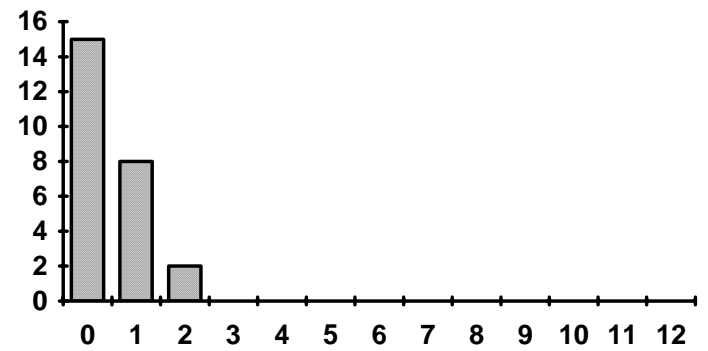

produçao

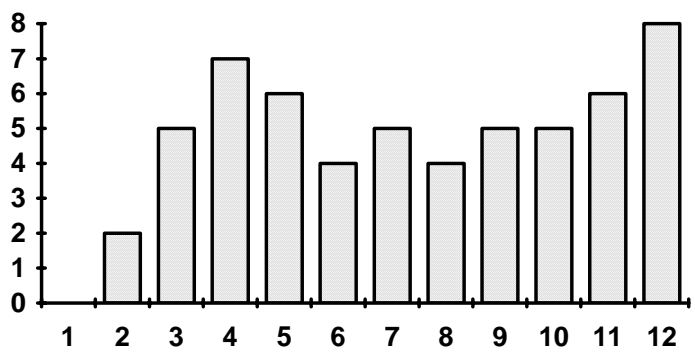

Figura 7.

Comentários: a análise dos três cenários mostra que o valor fornecido pelo usuário para afeta a solução ótima do problema. Assim, quanto menor for o valor de , maior será a área de segurança de estoque, gerada pela função restrição $\mathrm{r}_{\alpha}(\mathrm{k})$ e conseqüentemente maiores as chances de satisfação do cliente. Por outro lado, um preço pago para manter este estoque de segurança é o aumento do custo total de produção, ou seja: $\mathrm{J}_{0.5}^{*}<\mathrm{J}_{0.25}^{*}<\mathrm{J}_{0.05}^{*}$. Em particular, o cenário 3 mostra que se a política de produção, dada na figura 7 , for aplicada ao sistema produtivo, existirá o risco, em torno de $50 \%$, de não ser atendida a demanda em um dado período. Para que isso ocorra basta que a demanda real $\mathrm{v}(\mathrm{k})$ (que não é conhecida a priori) fique acima do nível médio de demanda $\overline{\mathrm{v}}(\mathrm{k})$, em algum período $\mathrm{k}$.

\section{CASO 3: Comparação de soluções}

Para aplicação do algoritmo de PD Estocástica ao problema (1), considera-se a demanda $\mathrm{v}(\mathrm{k})$, variando num intervalo que com-preende o mínimo de $\underline{\mathrm{v}}(\mathrm{k})=\overline{\mathrm{v}}(\mathrm{k})-2.58 * \sigma_{\mathrm{v}} \quad$ e $\quad$ o máximo de $\overline{\bar{v}}(\mathrm{k})=\overline{\mathrm{v}}(\mathrm{k})+2.58 * \sigma_{\mathrm{v}}$ Isto sig-nifica que o algoritmo trabalhará com a chance de mais de 95\% de ocorrência de um nível de demanda nesta faixa (CHOU, 1970). O grau de probabilidade fixado pelo usuário é de $=5 \%$. O estoque inicial considerado é $\mathrm{x}_{0}=15$. Os resultados obtidos estão ilustrados na figura 8 e os custos totais são: para a solução verdadeira $\mathrm{J}^{*}=1.351$ e para a solução aproximada $\mathrm{J}^{*}=2.019$.


Figura 8: Comparação entre a solução verdadeira ("verda”) e aproximada (“aprox”) para os níveis ótimos de estoque (“x”) e de produção (“u”). 
Comentário: É importante observar que a solução ótima verdadeira mantém um estoque de segurança para o problema (1), que é visível a partir do quarto período, ou seja, $\mathrm{x}(\mathrm{k})=4$, para $\mathrm{k}=4, \ldots, 12$. Isto mostra que em operações de longo prazo (> 1 ano) há a necessidade de manter-se um estoque regulador capaz de assegurar $\mathrm{o}$ atendi-mento da demanda flutuante. Comparando-se o estoque de segurança observado nas duas soluções, a partir do período $\mathrm{k}=2$, verifica-se que a área adicional criada pela solução aproximada é cerca de $60 \%$ maior. Isto pode ser interpretado como uma compensação devida às simplificações feitas para transformar (1) no equivalente determinístico (10). Em outras palavras, as incertezas sobre o comportamento futuro da demanda são projetadas na função limite $r_{\alpha}(k)$ que cresce proporcionalmente ao cres-cimento da variância de estoque $\sigma_{\mathrm{x}}(\mathrm{k})$, cuja evolução se dá sem nenhum

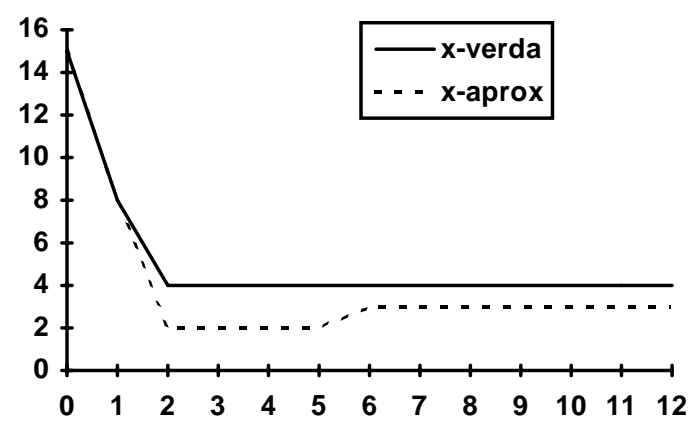

tipo de inter-ferência (i.e., controle) ao longo dos perío-dos de tempo (vide equação (3)). Este fato se reflete nos custos de operação; nota-se que, neste caso, o emprego da solução apro-ximada será 49\% mais caro que o da solu-ção verdadeira. O usuário pode, por meio de tentativas, melhorar a solução aproxi-mada em relação à solução verdadeira, bastando para isso aumentar gradativamen-te o valor de (como visto anteriormente no caso 2). Por exemplo, adotando-se $=0.25$ (i.e., $75 \%$ de chance de atendimen-to da demanda) obtém-se um custo ótimo de operação igual a $\mathrm{J}^{*}=1.386$ que é muito próximo do obtido para a solução verdadeira. As políticas ótimas de estoque e produção estão ilustradas na figura 9, na qual se pode observar que as trajetórias da solução aproximada estão bem mais ajustadas às trajetórias da solução verdadeira.



Figura 9.

\section{Conclusão}

Neste artigo discutiu-se a solução de um problema de planejamento ótimo da produção, com restrições de não-negatividade nos níveis de produção e de estoque, sendo que para este último a restrição é do tipo probabilística. Devido à complexidade deste problema, tanto em termos da dimensão (caso multiproduto) quanto da natureza estocástica, um problema determinístico equivalente é apresentado como uma alternativa viável para gerar políticas de produção aproximadas para o problema estocástico. No processo de conversão do problema original no seu equivalente determi- nístico foi observado que as incertezas decorrentes da flutuação de demanda são projetadas em uma função côncava crescente, que representa o limite mínimo de segurança para o nível de estoque. A área de segurança definida por esta função pode ser modificada pelo usuário a partir de uma medida de probabilidade por ele fornecida. Como conseqüência disto o usuário tem a seu dispor um mecanismo que lhe permite analisar várias possibilidades para o proble-ma e assim melhorar a solução final. Um exemplo simples permitiu verificar algumas das propriedades e 
condições apresentadas ao longo do trabalho e, além disso, possi-bilitou comparar a solução aproximada com a solução verdadeira obtida utilizando o algoritmo de programação estocástica.

Em termos de atuais pesquisas e desenvolvimentos realizados pelos autores nesta linha de trabalhos, alguns pontos que mere-cem ser destacados são: (1) a análise do problema em questão adotando uma mode-lagem mais abrangente, que seria consi-derar os níveis de estoque e de produção sujeitos a limites mínimos e máximos, respectivamente (SILVA Fo. et al., 1994); (2) considerar a possibilidade de adotar um esquema de solução do tipo malha-fechada, ou seja, o nível de produção dependente a cada período do nível de estoque. Neste caso, serão adotadas técnicas da Teoria de Controle Moderna; (3) estender o modelo apresentado em (1), de modo que ele considere outros tipos de custos e ainda adicionar à equação de balanço estoque/ produção, a variável nível de mão de obra; (4) outro importante ponto a ser destacado é o objetivo de integração deste modelo agregado de produção, no caso considerando várias famílias de produtos distintas, com um modelo para planejamento detalha-do (baseado em teoria de grafos), que vem sendo desenvolvido pelos autores e tem sido aplicado em uma empresa nacional de autopeças (CARVALHO et al., 1994).

\section{Agradecimentos:}

Esta pesquisa teve o apoio do CNPq, dentro do processo nº 501237/91-7 (NV).

\section{Referências Bibliograficas:}

AVRIEL, M.: Nonlinear Programming; Analysis and Methods. Prentice-Hall, Englewood Cliffs,1976.

BENSOUSSAN, A; CROUCHY, M. \& PROTH, J.M. (eds.): Mathematical Theory of Production Planning. NorthHolland, Amsterdam, 1983

BERTESEKAS, D. P.: Dynamic Programming and Stochastic Control. Academic Press, 1976

CARVALHO M.F.; FERNANDES, C.A. O.; SILVA Fo, O.S. \& VARGA Jr., E.J.: "OPERA - Optimal Production Environment for Resource Allocation”. IFIP Transactions B-19, pp 279288, North-Holland, 1994.

CHOU, Y.: Probability and Statistics for Decision Making. Holt, Rinehart and Wiston, Inc, New York, 1970

FEIRING, B.R. \& SASTRI, T.: "Improving Production Planning by Utilizing
Stochastic Programming”, Computers Ind. Eng., Vol. 19, pp 53-56, 1990.

HAX, A.C.: "Aggregate Production Planning". In: Morder, J. \& Elmaghraby, S.E (eds.).: Handbook of OR: Models and Applications (pp 127-169). Van Nostrand Reinhold Company, New York, 1978.

HILL, T.: Production/Operations Manage-ment: Text and Cases. Prentice Hall, 1991.

LASSERE, J.B.; BES, C. \& ROUBELLAT, F: "On the OpenLoop Solution of Linear Stochastic Control Problem", IEEE Trans. Automatic Control, vol. 29, no. 6, 1984.

LASSERE, J.B.; BES. C. \& ROUBELLAT, F.: "The Stochastic Discrete Dynamic Lot-Size Problem: An Open-loop Solution”. Operations 
Research, Vol. 3, No. 3, pp: 684-689, 1985.

NECK, R.: "Stochastic Control Theory and Operational Research". European Journal of Operational Research, v. 17, pp. 283-301, 1984.

PARLAR, M.: “A Stochastic Production Planning Model with a Dynamic Chance Constraint”. European Journal of Operational Research, 20, pp. 255260, 1985.
SILVA Fo. O.S.; CARVALHO, M.F. \& HENRIQUE, M.M.: "A Sub-optimal Policy for Stochastic Production Planning Problem". IFIP Transactions B-19, pp 297-304, North-Holland, 1994.

VADJA, S.: Probabilistic Programming. Academic Press, New York, 1972

\section{OPTIMAL AGGREGATE PRODUCTION PLANNING WITH MINIMUM INVENTORY LIMIT AFFECTED BY DEMAND UNCERTAINTIES}

\section{ABSTRACT:}

This paper deals with the determination of an optimal decision policy for a production planning problem with inventory and production constraints. The planning time horizon is finite, from 1 to 2 years approximately at monthly periods, which means that all data involved with the problem are totally aggregated and the fluctuating demand for each one period is stochastic, with probability distribution function assumed as gaussian. Thus the problem studied here is a stochastic planning one with probabilistic constraint at the inventory level variable. It is shown that it is possible to obtain, by means of appropriate transformations, a deterministic equivalent formulation, for which an open-loop solution to stochastic problems can be generated, using any applicable mathematical programming algorithm. It is also shown that the uncertainties concerning demand fluctuation are explicitly presented in the deterministic formulation through a constraint function that represents the minimum inventory level limit or safety stock. This function is essentially concave and increases during time and depends on the variance of inventory level and probabilistic degree supply by the user to the inventory constraint. To illustrate the theoretical developments, a simple example of a single product system is proposed and solved by dynamic programming. The open-loop solution (i.e. approximate solution from an equivalent problem) is compared with the true solution obtained directly from the stochastic problem via stochastic dynamic programming.

Key-words: production planning, stochastic process, dynamic programming 